\title{
Direct Cannulation of Ascending Aorta versus Standard Femoral Artery Cannulation in Acute Aortic Dissection Type A
}

\author{
Vassil Gegouskov, MD, PhD, ${ }^{1}$ Georgi Manchev, MD,${ }^{1}$ Vladimir Danov, MD, $\mathrm{PhD},{ }^{2}$ Georgi Stoitsev, $\mathrm{MD},{ }^{1}$ \\ Sergey Iliev, $\mathrm{MD}, \mathrm{PhD}^{3}$ \\ ${ }^{1}$ Department of Cardiac Surgery, St. Anna University Hospital, Sofia, Bulgaria; ${ }^{2}$ Department of Cardiac Surgery, and 3Department of \\ Propedeutics of Surgical Diseases, Medical University, Pleven, Bulgaria
}

\section{ABSTRACT}

Background: During surgery for ascending aortic dissection, the dissected ascending aorta itself has traditionally been rejected as a cannulation option. The purpose of this study is to prove that direct cannulation of the ascending aorta in patients operated for acute aortic dissection type A (AADA) is at least as effective and safe as classic femoral cannulation.

Methods and Results: Between September 2008 and January 2015, we operated on 117 patients with AADA through median sternotomy. Cannulation was accomplished in 32 cases $(27 \%)$ through the femoral artery (group A), and in 85 patients $(73 \%)$ through the dissected ascending aorta (group B). Moderate hypothermic circulatory arrest with bilateral antegrade cerebral perfusion was used in 108 patients $(92 \%)$. The mean time of circulatory arrest was 17 minutes (range: $9-52$ minutes). The 30 -day mortality rate was $22 \%$ (7 patients) in group A, and 18\% (15 patients) in group B $(P=$ not significant $)$. Temporary neurologic dysfunction (TND) including postoperative confusion, delirium, or agitation occurred in four patients (13\%) in group A, and four patients $(5 \%)$ in group B $(P=$ not significant). The incidence of permanent neurologic dysfunction (stroke) was $9 \%$ (3 patients) in group A and 3\% (3 patients) in group B.

Conclusions: The direct cannulation of the ascending aorta is a safe alternative for patients with AADA, offering the opportunity for antegrade cerebral perfusion. It is easy to perform, reliable, and associated with acceptable early results.

\section{INTRODUCTION}

Acute aortic dissection type A (AADA) remains one of the biggest challenges for most cardiac surgeons. It is still associated with a high mortality rate (12-33\%) despite continuous surgical and technical advances and improvements in

Received December 28, 2017; received in revised form March 20, 2018; accepted March 24, 2018.

Presented orally at the 23rd Annual Meeting of the Asian Society for Cardiovascular and Thoracic Surgery 2015, May 11-14, 2015, Hong Kong.

Correspondence: V. Gegouskov, M.D., Department of Cardiac Surgery, St. Anna University Hospital, 1, D. Mollov Str., Sofia 1781, Bulgaria; +359896-718226 (e-mail:vg1@arcor:de). postoperative care [Khaladj 2008a; Reece 2007; Mehta 2002]. A major cause of mortality and morbidity in AADA repair patients is cerebrovascular injury and organ malperfusion, which may be influenced by the arterial cannulation method. Thus, the method used may represent a main determinant of operative outcomes.

Consensus has not yet been reached about the best method of arterial cannulation for cardiopulmonary bypass (CPB) when dealing with AADA. Generally, there are three cannulation strategies that represent the surgical approach of most cardiac surgery centers worldwide, and each method has its own advantages and disadvantages [Gobolos 2014]. Traditionally, during the late " 80 s and early ' 90 s, the femoral cannulation was the "gold standard" in most institutions for cardiac surgery [Khaladj 2008c]. Since this technique is associated with retrograde cerebral embolization, propagation of dissection, and organ malperfusion, many surgeons switched to an alternative approach for arterial cannulation [Krüger 2012].

Cannulation of the axillary/subclavian artery has become more popular in the last ten years, during which the benefits of antegrade body perfusion have become clearer. However, it is more time-consuming and requires a more precise technique, which is not always feasible in the emergent intervention that AADA usually necessitates [Shimokawa 2008; Reece 2007]. In addition, particularly in small patients, shortage of pump flow may be encountered due to the small caliber of the artery.

The third method is the direct (or central) cannulation of the dissected ascending aorta. It involves the Seldinger technique, and was first described in 2003 by the Hannover group [Minatoya 2003]. For over five years, our institution has preferred the direct cannulation of the dissected ascending aorta via the Seldinger technique. In this paper we present the early results after surgery for AADA using central and femoral cannulation techniques.

\section{MATERIALS AND METHODS}

\section{Patients}

Between September 2008 and January 2015, 117 patients underwent emergency surgery for AADA. After obtaining approval of the institutional review board at the St. Anna University Hospital, the patients' data were analyzed retrospectively. The patients were divided into two groups on the basis of the initial site of cannulation. In group A (32 
Table 1. Preoperative Patient Data

\begin{tabular}{lccc}
\hline Parameter & Femoral group A & Central group B & $P$ \\
\hline No. of patients & $\mathrm{n}=32(27 \%)$ & $\mathrm{n}=85(73 \%)$ & \\
Age (years, median \& range) & $64.8(46-79)$ & $56.2(22-81)$ & .86 \\
Gender (M/F) & $24 / 8$ & $59 / 26$ & .074 \\
Marfan syndrome & $1(3 \%)$ & $7(8 \%)$ & .041 \\
Previous thoracic surgery & $1(3 \%)$ & $5(6 \%)$ & .89 \\
Preoperative malperfusion: & & & \\
$\quad$ Cerebral ischemia & $2(6 \%)$ & $(8 \%)$ & .77 \\
$\quad$ Critical limb ischemia (>2h) & $1(3 \%)$ & $3(4 \%)$ & .88 \\
Abdominal malperfusion & $1(3 \%)$ & $4(5 \%)$ & .86 \\
Pericardial effusion & $4(13 \%)$ & $16(19 \%)$ & .068 \\
Cardiogenic shock & $2(6 \%)$ & $11(13 \%)$ & .027 \\
Arterial Hypertension & $29(90 \%)$ & $71(84 \%)$ & .84 \\
Diabetes mellitus & $6(19 \%)$ & $18(21 \%)$ & .81 \\
Hyperlipidemia & $15(47 \%)$ & $46(54 \%)$ & .78 \\
Smoking & $28(88 \%)$ & $79(93 \%)$ & .73 \\
CAD & $3(9 \%)$ & $7(8 \%)$ & .85 \\
\hline
\end{tabular}

CAD indicates coronary artery disease.

patients, $27 \%$ ), cannulation was accomplished through the femoral artery, and in group B (85 patients, $73 \%$ ), through the ascending aorta. The two groups were compared regarding their preoperative characteristics, operative procedures, perioperative morbidities and mortality, and a follow-up analysis was initiated.

The preoperative data and intraoperative parameters were somewhat similar in both groups (Tables 1 and 2). Median age was 64.8 years (range: 46-79) in group A, versus 56.2 years (range: $22-81)$ in group $\mathrm{B}(P=.86)$. The patients were $75 \%$ male in group A, and $69 \%$ male in group B. The number of patients with Marfan syndrome was significantly higher in group B (7 (8\%) vs. 1 (3\%), P = .041). Because our institution has a large catchment area, some patients arrived at the hospital in a severely compromised general condition. In group $\mathrm{B}, 11$ patients $(13 \%)$ were in cardiogenic shock, versus $2(6 \%)$ in group $\mathrm{A},(P=.027)$ (Table 1$)$.

\section{Surgical Procedure}

After confirmation of the diagnosis of AADA (through CT scan, MRI and/or echocardiography), all patients were transferred to the operating room immediately. No patient was denied surgery, regardless of their preoperative status. Under standard general anesthesia, the ascending aorta and arch were exposed through midline sternotomy, following the "in-house" surgical protocol (Table 3 ). Both radial and right femoral artery pressure measurements were utilized for monitoring. After median sternotomy and systemic heparinization, we prepared for CPB. All cases with direct cannulation were operated by the same surgeon (VG). The site of arterial
Table 2. Intraoperative characteristics

\begin{tabular}{lccc}
\hline & $\begin{array}{c}\text { Femoral } \\
\text { Proup A }\end{array}$ & $\begin{array}{c}\text { Central } \\
\text { group B }\end{array}$ & $P$ \\
\hline No. of patients & $\mathrm{n}=32(27 \%)$ & $\mathrm{n}=85(73 \%)$ & \\
Operation time (minutes) & $324(181-$ & $297(164-$ & .77 \\
& $808)$ & $733)$ & \\
CPB time & $176(87-323)$ & $155(78-288)$ & .84 \\
Cross-clamp time & $143(57-225)$ & $123(44-207)$ & .78 \\
Circulatory arrest time & $31(22-47)$ & $27(9-73)$ & .86 \\
Aortic root surgery & & & \\
Supracommissural replacement & $18(56 \%)$ & $49(58 \%)$ & .91 \\
Aortic valve reconstruction & $7(22 \%)$ & $16(19 \%)$ & .68 \\
Bentall procedure & $7(22 \%)$ & $20(23 \%)$ & .74 \\
Aortic arch surgery & & & \\
Hemiarch replacement & $24(75 \%)$ & $57(67 \%)$ & .88 \\
Total arch replacement & $4(13 \%)$ & $9(11 \%)$ & .83 \\
Arch plus elephant trunk & $2(6 \%)$ & $7(8 \%)$ & .71 \\
Arch plus frozen elephant trunk & - & $2(2 \%)$ & - \\
CABG & $3(9 \%)$ & $7(8 \%)$ & .81 \\
Cross-over bypass (femoro-femoral) & $1(3 \%)$ & $2(2 \%)$ & .82 \\
\hline
\end{tabular}

CPB indicates cardiopulmonary bypass; CABG, coronary artery bypass graft.

cannulation was decided individually based on the surgeon's preference. The femoral cannulation was done via an open technique. The artery was exposed, a transverse arteriotomy was made, and a Medtronic EOPA® arterial cannula 20 Fr was inserted and snared (Figure 1).

Since 2012, the dissected ascending aorta or arch has been routinely cannulated using the Seldinger method under transoesophageal ultrasound guidance. When looking for an area where the aortic wall appears to be intact, the ascending aorta was inspected, and the intraoperative findings were compared with those of the CT scan and the transesophageal echo. The pulmonary side of the ascending aorta and the concavity of the arch are commonly spared by the dissection process. This

Table 3. “In-House” Surgical Protocol for AADA.

\footnotetext{
1. Pressure monitoring with 3 arteries (1 FA $+2 \mathrm{RA})$

2. Systemic heparinization prior to sternotomy.

3. Cannulation of the right atrium FIRST.

4. Direct Cannulation of the dissected aorta.

5. Systemic cooling to $26-30^{\circ} \mathrm{C}$.

6. Open distal anastomosis with bilateral SACP.

7. Rewarming with max. temperature gradient of $6-8^{\circ} \mathrm{C}$.
}

FA indicates femoral artery; RA, radial artery; SACP, selective antegrade cerebral perfusion. 


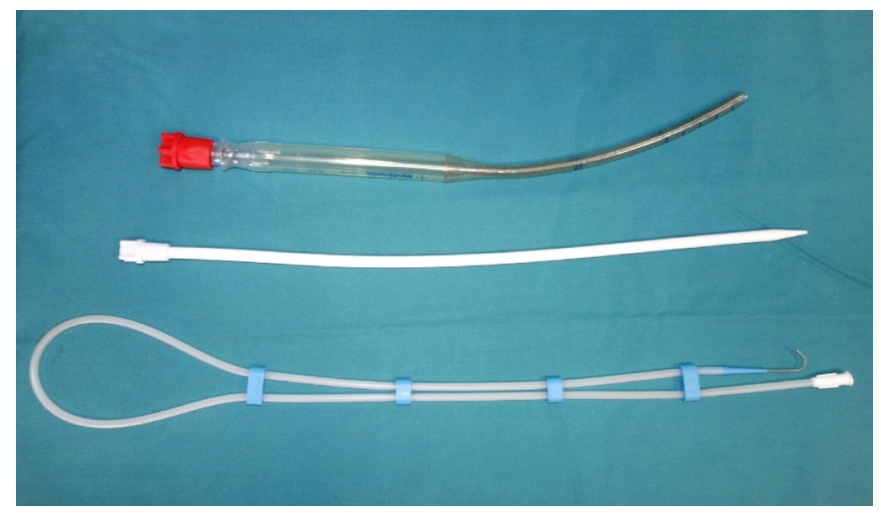

Figure 1. Arterial cannula for central cannulation.

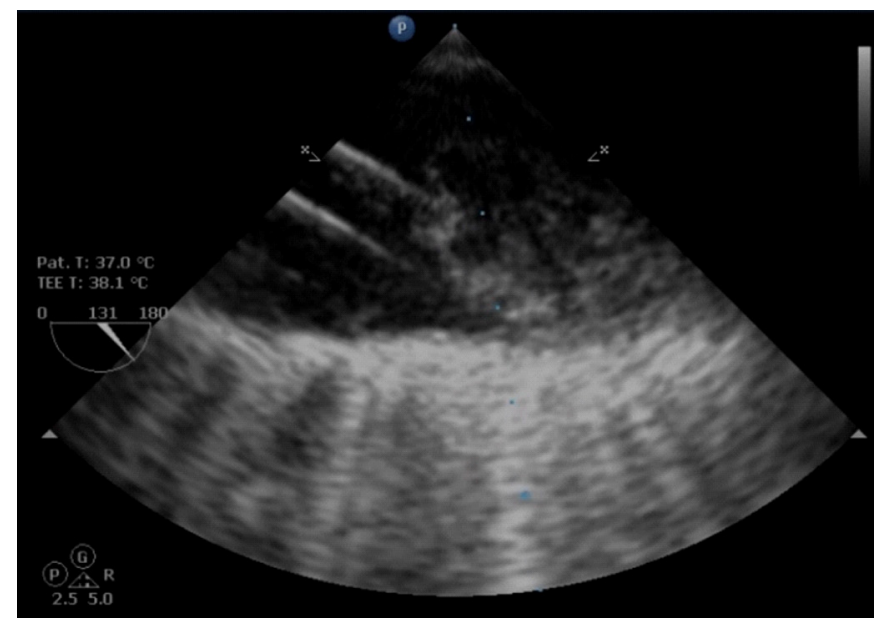

Figure 2. Transesophageal echocardiogram (TEE) visualization of perfusion of the true lumen.

inner arch segment contains more connective tissue, which makes it more resistant to tear. After vascular puncture, the guidewire was inserted and its position in the true lumen was echocardiographically confirmed (Figure 2). Following the careful insertion of the wire-wound Medtronic EOPA® arterial cannula 20 Fr. with a tapered introducer (Medtronic, Inc., Minneapolis, MN, USA), its position was checked again, visualizing the perfusion of the true lumen of the aorta (Figure 1). Finally, the cannula was secured with one purse-string suture and was fixed with a stitch to the upper skin incision.

After this, CPB was started with core cooling. The ascending aorta was incised after cross-clamping, and cold crystalloid cardioplegia (Custodiol@ HTK solution) was infused through both coronary orifices. Root reconstruction was then performed as necessary. When the target bladder temperature was reached (26-30 $\left.{ }^{\circ} \mathrm{C}\right), \mathrm{CPB}$ was stopped, the aorta was opened, and the cannula removed. In 92\% (108 patients) of cases, open distal anastomoses with bilateral selective antegrade cerebral perfusion (bSACP) (84\% in the femoral group and $95 \%$ in the central group) were performed, using the Medtronic 15 Fr DLP® cannula for retrograde cardioplegia with manual-inflation ridged cuff (Medtronic, Inc., Minneapolis, MN, USA) (Figure 3).

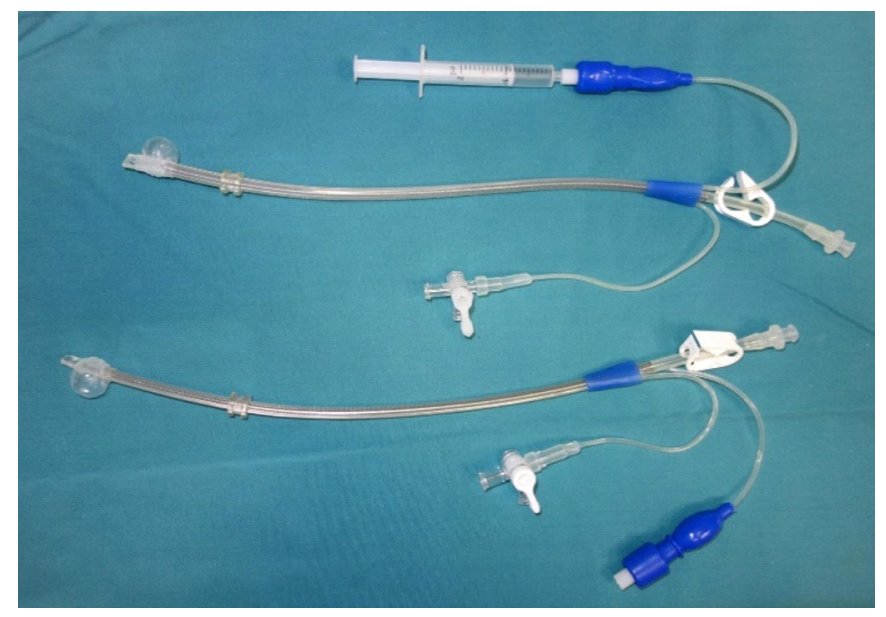

Figure 3. Retrograde cardioplegia cannula (12 or $15 \mathrm{Fr}$ ) for selective antegrade cerebral perfusion (SACP).

Cerebral blood flow was maintained at $10 \mathrm{~mL} / \mathrm{kg} / \mathrm{min}$ at a perfusion pressure of $50-60 \mathrm{mmHg}$ in order to keep stable brain oxygen saturation. Cerebral monitoring was performed with near-infrared spectroscopy (INVOS® cerebral oximeter, Medtronic, Minneapolis, MN, USA). The head was additionally cooled with ice packs during the arrest period. Pharmacological protection of the brain was comprised of $1 \mathrm{~g}$ of methylprednisolone and $1 \mathrm{~g}$ of thiopental before circulation was stopped. The mean time of circulatory arrest was $17 \mathrm{~min}$ utes (range: 9-52). The extent of the surgical procedure was dependent on whether re-entry tears were found in the arch.

\section{Statistical analysis}

The collected data were statistically analyzed using SPSS 14.0 software (SPSS Inc., Chicago, IL, USA). The results were expressed as mean values \pm standard deviation (SD), and median plus range or percentage. For univariate analysis, contingency table analysis for dichotomous and chi-square tests were used. Odds ratios for categorical variables and chisquare analysis for the strength of association between variables were performed.

\section{Results}

No intraoperative deaths occurred. Cumulative 30-day mortality rate was $18.8 \%$ for the entire cohort, $22 \%$ ( 7 patients) in the femoral group, and $18 \%$ (15 patients) in the central group $(P=.94)$. The direct aortic cannulation was successful in all cases, and there was no need to switch to an alternative cannulation site. The incidence of permanent neurological dysfunction (stroke) was $9 \%$ (3 patients) in group A and 3\% (3 patients) in group B; all patients suffering stroke succumbed $(P=0.87)$. Other causes of death are displayed in the table 4. Temporary neurological dysfunction (TND) including postoperative confusion, delirium, or agitation occurred in four patients $(13 \%)$ in group $\mathrm{A}$ and four patients $(5 \%)$ in group $\mathrm{B}(P=.82)$. All these patients recovered completely before discharge.

All nine patients with preoperative cerebral ischemia underwent selective antegrade cerebral perfusion (SACP). 
Table 4. Follow-Up Results

\begin{tabular}{lccc}
\hline Parameter & $\begin{array}{c}\text { Femoral } \\
\text { group A }\end{array}$ & $\begin{array}{c}\text { Central } \\
\text { group B }\end{array}$ & $P$ \\
\hline No. of patients & $\mathrm{n}=32(27 \%)$ & $\mathrm{n}=85(73 \%)$ & \\
30-day mortality & $7(22 \%)$ & $15(18 \%)$ & .94 \\
Morbidity & & & \\
Central malperfusion (stroke) & $3(9 \%)$ & $3(3 \%)$ & .87 \\
TIA & $2(6 \%)$ & $5(6 \%)$ & .82 \\
Temporary postoperative confusion & $4(13 \%)$ & $4(5 \%)$ & .54 \\
Peripheral malperfusion & $2(6 \%)$ & $6(7 \%)$ & .86 \\
Re-exploration for bleeding & $2(6 \%)$ & $4(4 \%)$ & .77 \\
Renal failure & $3(9 \%)$ & $7(8 \%)$ & .69 \\
Multiorgan dysfunction & $1(3 \%)$ & $3(4 \%)$ & .84 \\
Sepsis & $1(3 \%)$ & $2(2 \%)$ & .88 \\
\hline
\end{tabular}

TIA indicates transient ischemic attack.

These patients had expectedly higher rate of postoperative stroke (PS). The odds ratio for developing PS is 17.5 for patients not receiving SACP compared to those receiving SACP. The relative risk for PS was 12 times higher when no SACP was employed. The association between SACP and permanent neurologic injury was statistically significant $(P=.001)$, but it was weak to moderate $(\varphi=.369)$. When patients with preoperative neurological symptoms were excluded, the positive influence of SACP on postoperative stroke was even stronger. The relative risk for stroke rose to 33 when no cerebral perfusion was utilized $(P=.001)$.

If the patients with no protective brain perfusion were ignored, the odds for developing PS were 6.4 times greater in the femoral group than the central group. The relative risk for stroke in the femoral group was six. However, the association between the cannulation method and the occurrence of PS was weak $(\varphi=.163)$, and not statistically significant $(P=.091)$. When patients with preoperative cerebral ischemia were excluded from analysis, then the odds for PS were 2.7 for the femoral arm. The association, however, did not reach statistical significance $(P=.312)$, and the association was weak $(\varphi=.097)$.

More impressive results were obtained when the effect of SACP on TND (transient ischemic attack and delirium) was assessed. Cases with neurological symptoms on presentation were omitted. The odds for TND were 64 when no SACP was used during surgery. The association was statistically significant $(P=.000)$ and it was fairly strong $(\varphi=.64)$.

Reopening the chest for bleeding was necessary in $6 \%$ ( 2 patients) in the femoral group, and in 5\% (4 patients) in the central group. Postoperative renal failure (defined as the need for temporary renal replacement therapy) developed in three patients (9\%) from group A, and seven patients (8\%) from group B. Malperfusion was recognized as a drop in the cerebral oxygen saturation, a pressure difference between the left and right radial arteries, or a sudden pressure elevation in the arterial cannula [Kamiya 2009]. Mean operative times, CPB duration, cross-clamp times, and circulatory arrest times were shorter in the central group, although the differences did not reach statistical significance. Operative corrections to the root and aortic arch were comparable between the two groups and are shown in Table 2.

\section{DISCUSSION}

Femoral and axillary arteries used to be the most popular cannulation sites for arterial inflow in AADA in current practice. Central cannulation of the dissected aorta has been viewed with reluctance due to the fragile nature of the dissected aorta, the possibility for false lumen cannulation and various malperfusion phenomena, the risk of aortic rupture on commencement of CPB, and propagation of the dissection. Many studies, though, report no such complications, and neither do we [Conzelmann 2009; Khaladj 2008b; Khaladj 2008c]. So far, with 85 patients we have not experienced local complications at the aortic cannulation site. In addition, ascending aortic cannulation may be especially beneficial due to its technical simplicity in the event of pericardial tamponade and hemodynamic instability. In this way, it may prevent longer periods of shock and decrease mortality.

As experience with conventional cannulation strategies grows, it becomes evident that they bear their own risks and imperfections. Although in our group we had no perfusion complications, femoral cannulation is associated with a higher risk of retrograde brain embolization, and potential perfusion of the false lumen through a distal re-entry tear [Shimokawa 2008; Robicsek 1985]. Femoral cannulation in the setting of peripheral vascular disease may lead to malperfusion and arterial injury. Despite this, other groups report acceptable results with femoral cannulation [Fusco 2004]. Antegrade perfusion methods may obviate such risks associated with femoral cannulation. Luciani et al report a method of antegrade body perfusion with a long cannula through the femoral artery [Luciani 2012]. Axillary/subclavian artery inflow ensures antegrade body perfusion and unilateral brain perfusion during the arrest period, and is described as the optimal cannulation approach by some groups [Flege 2001]. A fraction of the population may have an incomplete circle of Willis, and thus only one cerebral hemisphere remains perfused [Papanchev 2013; Merkkola 2006]. Axillary/subclavian access requires more time for preparation, more precise technique, and may be apt to limited pump flow especially in patients with a small body surface area [Sinclair 2003]. Local ischemia or hyperemia of the arm is also a recognized complication [Sinclair 2003].

Frederick et al developed a useful anatomic classification of ascending aortic dissection based on the expected difficulty of cannulating the true lumen via the Seldinger technique [Frederick 2013]. They describe three types of dissection with increasing levels of difficulty for successful cannulation.

Direct cannulation of the ascending aorta is rapid and straightforward, though it may result in complete aortic rupture, false lumen perfusion, and propagation of the dissection. 
However, our study and others demonstrate that central cannulation is comparable or even superior to the conventional femoral site when treating AADA [Gobolos 2014]. The Hannover group were the first to describe their experience with central cannulation, and then extended their results [Khaladj 2008c; Minatoya 2003]. Other authors have also concluded that femoral and ascending aortic cannulation in the treatment of AADA provide comparable results [Conzelmann 2016; Klotz 2016; Etz 2014; Suzuki 2010]. Nevertheless, some studies reveal significantly higher mortality rates in peripheral cannulation groups [Kamiya 2009; Reece 2007]. Shorter operative times may indicate the improvement of technical skills and rapidity of the operating surgeon because we started to use this arterial approach more recently, but nevertheless, central cannulation takes the least time to perform compared to all peripheral methods.

Ultrasonographic verification of correct cannula position is mandatory since one cannot be sure of true lumen cannulation, because both lumens are well perfused in most cases. Since antegrade true lumen perfusion is established, we are comfortable with cross-clamping the aorta. This allows aortic valve and root reconstruction during the cooling phase, thus reducing the total CPB duration. Cerebral oximetry is essential in all AADA repair cases as it may alert the operative team of potential brain injury before it occurs, and trigger appropriate measures such as repositioning the arterial cannula or change of the cannulation site.

Postoperative neurological complications, whether permanent or transient, appeared with near equal frequencies in both cannulation groups. Other investigators have shared the same results in their series [Klotz 2016; Kamiya 2009].

Despite surgical and technical advances, mortality rates after treatment of acute AADA remain high and in a wide range (12-33\%) [Klotz 2016; Khaladj 2008a; Reece 2007; Mehta 2002]. Our single-center mortality rates in 117 patients are acceptable $(18.8 \%)$ in view of the fact that we did not reject any patients, no matter what their neurological or general status was. However, the cannulation method had no impact on mortality in our cohort. Some authors, however, report lower mortality rate with the central cannulation technique [Kamiya 2009].

We currently perform bSACP in virtually every case as it allows more moderate hypothermia, which shortens bypass times and diminishes coagulopathy. The superiority of brain perfusion over no perfusion with deep hypothermia has been proven by many studies [Khaladj 2006; Hagl 2004]. Central cannulation is not associated with an increased risk of cerebral microemboli [Kamiya 2007; Kamiya 2006]. The safety of direct aortic cannulation in AADA patients is confirmed by many investigators [Gobolos 2014; Conzelmann 2009; Kamiya 2009; Shimokawa 2008; Jakob 2007].

Two authors describe an alternative approach to ascending aortic cannulation. They transect the aorta, insert a straight arterial cannula directly into the true lumen under visual control, and snare the aorta with a tape or clamp it [Conzelmann 2009; Jakob 2007]. The authors believe that accidental cannulation of the false lumen with the consequent dispersion of thrombotic material is thus prevented.

\section{LIMITATIONS}

This is a single institution retrospective analysis in a heterogeneous population. Patients were not randomized as to which cannulation strategy is to be used. The choice of the cannulation method was based on surgeon's preference and experience. Thus, patient selection and surgeon preference bias were inevitable in this study. In addition, our cohort was relatively small and showed statistically significant differences.

\section{CONCLUSIONS}

Cannulation of the dissected aorta is safe enough to be used as a routine method for repair of AADA with results comparable or superior to other cannulation strategies. It is easy to perform, reliable and associated with acceptable early results.

\section{REFERENCES}

Conzelmann L, Kayhan N, Mehlhorn U et al. 2009. Reevaluation of direct true lumen cannulation in surgery for acute type a aortic dissection. Ann Thorac Surg 87:1182-6.

Conzelmann L, Weigang E, Mehlhorn U et al. 2016. Mortality in patients with acute aortic dissection type a: analysis of pre- and intraoperative risk factors from the german registry for acute aortic dissection type A (GERAADA). Eur J Cardiothorac Surg 49:e44-e52.

Etz CD, von Aspern K, da Rocha E Silva J, et al. 2014. Impact of perfusion strategy on outcome after repair for acute type a aortic dissection. Ann Thorac Surg 97:78-85.

Flege JB, Aberg T. 2001 Transventricular aortic cannulation for repair of aortic dissection. Ann Thorac Surg 72:955-6.

Frederick J, Yang E, Trubelja A et al. 2013. Ascending aortic cannulation in acute type a dissection repair. Ann Thorac Surg 95:1808-11.

Fusco DS, Shaw RK, Tranquilli M et al. 2004. Femoral cannulation is safe for type a dissection repair. Ann ThoracSurg 78:1285-9.

Gobolos L, Ugocsal P, Foltan M et al. 2014. Central cannulation by seldinger technique: a reliable method in type a aortic dissection repairs. Med Sci Monit 20: 2386-93

Hagl C, Khaladj N, Peterss S et al. 2004. Hypothermic circulatory arrest with and without cold selective antegrade cerebral perfusion: impact on neurological recovery and tissue metabolism in an acute porcine model. Eur J Cardiothorac Surg 26:73-80.

Jakob H, Tsagakis K, Szabo A et al. 2007. Rapid and safe direct cannulation of the true lumen of the ascending aorta in acute type a aortic dissection. J Thorac Cardiovasc Surg 134:244-5.

Kamiya H, Hagl C, Kropivnitskaya I et al. 2007. The safety of moderate hypothermic lower body circulatory arrest with selective cerebral perfusion: a propensity score analysis. J Thorac Cardiovasc Surg 133: 501-9.

Kamiya H, Kallenbach K, Halmer D et al. 2009. Comparison of ascending aorta versus femoral artery cannulation for acute aortic dissection type a. Circulation. 120 [suppl 1]:282-6.

Kamiya H, Klima U, Hagl C et al. 2006. Cerebral microembolization during antegrade selective cerebral perfusion. Ann Thorac Surg 81:519-21.

Khaladj N, Peterss S, Oetjen P et al. 2006. Hypothermic circulatory arrest with moderate, deep or profound hypothermic selective antegrade cerebral perfusion: which temperature provides best brain protection? Eur J Cardiothorac Surg 30:492-8.

Khaladj N, Shrestha M, Meck S et al. 2008. Hypothermic circulatory 
arrest with selective antegrade cerebral perfusion in ascending aortic and aortic arch surgery: A risk factor analysis for adverse outcome in 501 patients. J Thorac Cardiovasc Surg 135:908-14.

Khaladj N, Shrestha M, Peterss S et al. 2008. Ascending aortic cannulation in acute aortic dissection type a: the Hannover experience. Eur J Cardiothorac Surg 34:792-7.

Khaladj N, Shrestha M, Peterss S et al. 2008. Ascending aortic cannulation in acute aortic dissection type A: the Hannover experience. Eur J Cardiothorac Surg. 34:792-6.

Klotz S, Bucsky B, Richardt D et al. 2016. Is the outcome in acute aortic dissection type a influenced by femoral versus central cannulation? Ann Cardiothorac Surg 5:310-16.

Krüger T, Conzelmann LO, Bonser RS, et al. 2012. Acute aortic dissection type a. Br J Surg 99:1331-44.

Luciani N, Anselmi A, Glieca F et al. 2012. Femoral cannulation with long arterial cannula in aortic dissection. Ann Thorac Surg 93:e45-7.

Mehta RH, Suzuki T, Hagan PG et al. 2002. Predicting death in patients with acute type a aortic dissection. behalf of the international registry of acute aortic dissection (IRAD) investigators. Circulation 105:200-6.

Merkkola P, Tulla H, Ronkainen A et al. 2006. Incomplete circle of willis and right axillary artery perfusion. Ann Thorac Surg 82:74-9.
Minatoya K, Karck M, Szpakowski E et al. 2003. Ascending aortic cannulation for stanford type a acute aortic dissection: another option. J Thorac Cardiovasc Surg 125:952-3.

Papanchev V, Stoinova V, Aleksandrov A et al. 2013. The role of willis circle variations during unilateral selective cerebral perfusion: a study of 500 circles. Eur J Cardiothorac Surg 44:743-53.

Reece TB, Tribble CG, Smith RL et al. 2007. Central cannulation is safe in acute aortic dissection repair. J Thorac Cardiovasc Surg 133:428-34.

Robicsek F, Guarino RL. 1985. Compressing of the true lumen by retrograde perfusion during repair of aortic dissection. J Cardiovasc Surg 26:36-40.

Shimokawa T, Takanashi S, Ozawa N et al. 2008. Management of intraoperative malperfusion syndrome using femoral artery cannulation for repair of acute type a aortic dissection. Ann Thorac Surg 85:1619-24.

Sinclair MC, Singer RL, Manley NJ et al. 2003. Cannulation of the axillary artery for cardiopulmonary bypass: safeguards and pitfalls. Ann Thorac Surg. 75:931-34.

Suzuki T, Asai T, Matsubayashi K et al. 2010. Safety and efficacy of central cannulation through ascending aorta for type a aortic dissection. Interact Cardiovasc Thorac Surg 11:34-37. 